\title{
Hysteresis models and gravity fingering in porous media
}

\author{
Andreas Rätz and Ben Schweizer \\ Preprint 2012-02 \\ März 2012
}

Fakultät für Mathematik

Technische Universität Dortmund

Vogelpothsweg 87

44227 Dortmund 



\title{
Hysteresis models and gravity fingering in porous media
}

\author{
Andreas Rätz \& Ben Schweizer ${ }^{1}$
}

March 16, 2012

\begin{abstract}
We study flow problems in unsaturated porous media. Our main interest is the gravity driven penetration of a dry material, a situation in which fingering effects can be observed experimentally and numerically. The flow is described by either a Richards or a two-phase model. The important modelling aspect regards the capillary pressure relation which can include static hysteresis and dynamic corrections. We report on analytical existence and instability results for the corresponding models and present numerical calculations. We show that fingering effects can be observed in various models and discuss the importance of the static hysteresis term.
\end{abstract}

key-words: two-phase flow, hysteresis, gravity fingering instability

subject classification: $76 \mathrm{~S} 05$

\section{Introduction}

Gravity driven flow of water into an initially dry porous material can lead to fingering effects. This fact coincides with our elementary intuition, and the fingering effect can be observed experimentally, see e.g. $[2,9,16]$. On the other hand, standard mathematical models for flow in porous media such as Richards equation or the two-phase flow system cannot explain the fingering effect. This is due to stability properties of these equations, unless hysteresis or dynamic terms are included, see e.g. $[4,11,18]$.

Fingering can be observed in mathematical models only when such additional terms are incorporated. One term is related to the rate independent hysteresis that is observed when imbibition and drainage processes are performed after each other. The other term is related to non-equilibrium effects and is rate dependent. We investigate here both contributions in the capillary pressure relation. To emphasize the distinction between both effects, we sometimes call the rate independent term "static hysteresis"; it can be physically explained with the bottle-neck effect in the single pores. The other contribution is a dynamic term, often called the $\tau$-term, in reference to the standard name associated to the corresponding time-scale variable.

\footnotetext{
${ }^{1}$ Technische Universität Dortmund, Fakultät für Mathematik, Vogelpothsweg 87, D-44227 Dortmund, Germany.
} 
This term is sometimes denoted as "dynamic hysteresis", which is a useful terminology, even though it is mathematically not precise, since hysteresis is by definition rate-independent. We investigate here the extended porous media model with static and dynamic hysteresis as it has been introduced in [3] and mention that another hysteresis model has been studied in [1].

Our study extends previous results of $[7,8,15]$. In [8], we have shown analytically that the full hysteresis model is well-posed for the Richards equation, and we have shown the well-posedness for the two-phase flow model in [7]. In [15], we have shown rigorously that the Richards equation with static hysteresis does not define an $L^{1}$-contraction. In particular, we have shown that an instability occurs for these equations, making fingering possible. Numerical experiments show that fingers can indeed be observed. An important point that had been left out in these previous studies regards the relevance of static hysteresis. With the contribution at hand, we show the importance of static hysteresis: dynamic terms alone cannot describe fingering very well, but, instead, the combined effect of static and dynamic hysteresis leads to satisfactory results.

\subsection{Flow in porous media}

We start with a description of the flow models. We use $\Omega \subset \mathbb{R}^{n}$ as domain of porous material, the flow is time dependent and we use $t \in[0, T)$ as a time parameter. The pressures of the two fluids are $p_{1}, p_{2}: \Omega \times[0, T) \rightarrow \mathbb{R}$, the saturation of the first fluid is $s=s_{1}: \Omega \times[0, T) \rightarrow \mathbb{R}$, the saturation of the second fluid is $s_{2}=1-s_{1}=1-s$. If we combine Darcy's law for both velocities with conservation of mass, we obtain the two-phase flow equations

$$
\begin{aligned}
\partial_{t} s & =\nabla \cdot\left(k_{1}(s)\left[\nabla p_{1}+g_{1}\right]\right), \\
-\partial_{t} s & =\nabla \cdot\left(k_{2}(s)\left[\nabla p_{2}+g_{2}\right]\right),
\end{aligned}
$$

where $g_{1}$ and $g_{2}$ are related to gravity forces. We emphasize that quantities such as porosity or density are normalized in these equations, and that the permeabilities $k_{1}(s)=k_{1}(s(x, t), x)$ and $k_{2}(s)=k_{2}(s(x, t), x)$ are described by given functions $k_{1}, k_{2}$ : $[0,1] \times \Omega \rightarrow[0, \infty)$.

Starting from the two-phase flow equations, we obtain the Richards equation as the simplification where the second fluid is not modelled. This means that we assume $p_{2}$ to be constant, with a normalization we may assume $p_{2} \equiv 0$. Abbreviating $k(s):=k_{1}(s), p:=p_{1}$, and $g:=g_{1}$, equation (1.1) reduces to the Richards equation

$$
\partial_{t} s=\nabla \cdot(k(s)[\nabla p+g]) .
$$

An interesting feature of both systems, the two-phase flow system and the Richards equation, is the degeneracy of the coefficient functions. In applications one is interested in models with $k_{1}(0)=0$. A vanishing permeability makes the system degenerate, and a priori estimates are not available in standard function spaces. This makes existence proofs intricate even in models without hysteresis. We mention that small values of the permeability turned out to be important in numerical experiments, we could observe the generation of fingers only in settings with small permeabilities. Nevertheless, in the following, we neglect this degeneracy and concentrate on the hysteresis effects that enter the capillary pressure relation. 


\subsection{Capillary pressure relation}

Much of current research is related to the modelling problem regarding the relation between the capillary pressure $p_{1}-p_{2}$ and the saturation $s$. The simplest possibility is to assume a (pointwise) algebraic relation $p_{1}-p_{2}=p_{c}(s)$, where $p_{c}: \mathbb{R} \rightarrow \mathbb{R}$ (or $p_{c}:\left[s_{A}, s_{B}\right] \rightarrow \mathbb{R}$ or $\left.p_{c}:\left(s_{A}, s_{B}\right) \rightarrow \mathbb{R}\right)$ is a given function. In this case, the coefficient function $p_{c}$ is called, with full rights, the capillary pressure function. The problem in the assumption of an algebraic relation is that it can neither distinguish between imbibition and drainage, nor does it take non-equilibrium effects into account.

Measurements in porous media show hysteresis curves in experiments with imbibition and drainage. Since the typical hysteresis curves are measured also in experiments with extremely low rates, we include a rate-independent hysteresis term in the model. The simplest hysteresis law is that of play-type hysteresis, which can be written as

$$
p_{1}-p_{2} \in p_{c}(s)+\gamma \operatorname{sign}\left(\partial_{t} s\right),
$$

where sign $: \mathbb{R} \rightarrow \mathbb{R}$ is the multi-valued sign-function with $\operatorname{sign}(0)=[-1,1]$. The law (1.4) appears e.g. in [3] and can also be justified with a pore-scale analysis in a simplified pore geometry, see $[13,14]$.

Even though, in first approximation, a rate independent law seems to be an appropriate model for the capillary pressure relation, the discussion of non-equilibrium Richards equations shows that rate-dependent terms can also be relevant, compare e.g. $[6,10]$. The most prominent model uses a factor $\tau>0$, which can be understood as a relaxation time in the saturation law. This $\tau$-model is described in [3], where it is also combined with play-type hysteresis to the relation

$$
p_{1}-p_{2} \in p_{c}(s)+\gamma \operatorname{sign}\left(\partial_{t} s\right)+\tau \partial_{t} s .
$$

Even without static hysteresis, a positive parameter $\tau>0$ leads to interesting effects. For $\gamma=0$, it introduces a term with first time derivative and second spatial derivatives, thus turning the Richards equation into a pseudo-parabolic equation. Even in the limit of a vanishing influence of the capillary pressure, it may change the profile of travelling wave solutions, see [17]. It is well-known that a positive $\tau$ can lead to non-monotonic profiles in wetting fingers, [5].

We note that the monotone graph $\Xi: \xi \mapsto \gamma \operatorname{sign}(\xi)+\tau \xi$ with a vertical part can be inverted as a graph. We obtain a monotone graph which is actually a function, $\Psi_{\gamma, \tau}:=\Psi:=(\Xi)^{-1}: \mathbb{R} \rightarrow \mathbb{R}$, containing a flat part. The inverse allows to write relation (1.5) pointwise as an ordinary differential equation,

$$
\partial_{t} s=\Psi_{\gamma, \tau}\left(p_{1}-p_{2}-p_{c}(s) ; x\right) .
$$

Hysteresis enters by the fact that $\Psi$ has a flat part. The extra parameter $x \in \Omega$ indicates that in relation (1.5) the non-negative material parameter $\gamma=\gamma(x)$ may depend on the spatial position. An explicit spatial dependence may also be introduced in the capillary pressure function $p_{c}(s)=p_{c}(s, x)$.

\subsection{Capillary hysteresis leading to instability and fingering}

An important observation regarding static hysteresis in fingering experiments is the following. During a pure wetting process, only increasing saturation values appear; in 
consequence, the contribution of the hysteresis term is $\gamma \operatorname{sign}\left(\partial_{t} s\right)=\gamma$, it contributes only the additive constant $\gamma$ to the pressure in either (1.4) or (1.5). Therefore, in processes where imbibition occurs everywhere and at all times, static hysteresis does not alter the time evolution of the saturation.

The observation seems to make static hysteresis meaningless in fingering processes, but we must make two additional observations. One is related to the experimental set-up of fingering experiments. Usually, in a first part of the experiment, a region of high saturation is generated in a thin top layer of the material, this is done, say, from time $t=t_{0}<0$ until time $t=t_{s}=0$. At the "switching time" $t_{s}$, the boundary conditions are changed and the experiment is run with a smaller inflow rate or with a lower pressure at the top boundary. This means that a drainage process happens in the pre-wetted top layer for positive times, $t>t_{s}=0$.

A second observation regards the combined effect of the dynamic term and static hysteresis. A positive factor $\tau$ can create non-monotone profiles in the fingers. This indicates that a drainage process may occur also inside the fingers and thus static hysteresis can make a crucial difference. We will see this fact in our one-dimensional examples.

\section{One-dimensional examples}

In Figures 1 and 2 we show four sets of graphs. They illustrate saturation distributions $x \mapsto s(x, t)$ and pressure distributions $x \mapsto p(x, t)$ for fixed time instances $t$ in the one-dimensional case. All graphs illustrate the Richards equation. The four graphs illustrate the four different hysteresis models that have implicitly been introduced above: (1) no hysteresis, $\gamma=0$, and no dynamic term, $\tau=0$, in Figure 1 , left. (2) $\gamma>0$ and $\tau=0$, in Figure 1, right. (3) $\gamma=0$ and $\tau>0$, in Figure 2, left. (4) $\gamma>0$ and $\tau>0$, in Figure 2, right.

All models are calculated with a sudden change in the upper boundary condition. At time $t_{s}=0$, the influx condition on the upper boundary $x=12$ is switched from a higher value to a lower value. In all models, the switching process induces a decrease of pressure in a region near the upper boundary. In the models with static hysteresis, pressure and saturation are no longer in the algebraic imbibition relation in this upper region (the right region in the figures).

We used a fixed Dirichlet boundary condition $p=1$ at the lower boundary $x=$ -12 and an initial condition $s\left(t_{0}\right) \equiv 0$ for the initial time $t_{0}=-4$. The permeability $k=k(s)$ is given by (4.2) with parameters $\kappa=\kappa_{1}=0.1, a=a_{1}=0.35$, and $k_{0,1}=1$. We have chosen the regularizing parameter $\delta=10^{-5}$ in (4.1), which is used to approximate the hysteresis relation (1.6).

\subsection{Without dynamic term, $\tau=0$}

For the configuration of Figure 1, right, the Richards equation with static hysteresis and switching in the boundary condition, we have shown an instability result in [15]. With the gravity term, the system possesses unstable front solutions. Such an instability is the basis for fingering effects. 
The arguments for the instability are as follows. In the first process, the wetting of the top layer in the time interval $\left[t_{0}, t_{s}\right]$, the system is dissipative and small deviations of the saturation distribution have the tendency to be smeared out. Nevertheless, due to hysteresis and the lower pressure values after time $t=t_{s}$, all deviations from a uniform saturation distribution at time $t=t_{s}$ remain present for all later times. This means that imperfections in the wet top layer, e.g. non-homogeneity in horizontal spatial directions, are not wiped out, and they influence the process for all later times.
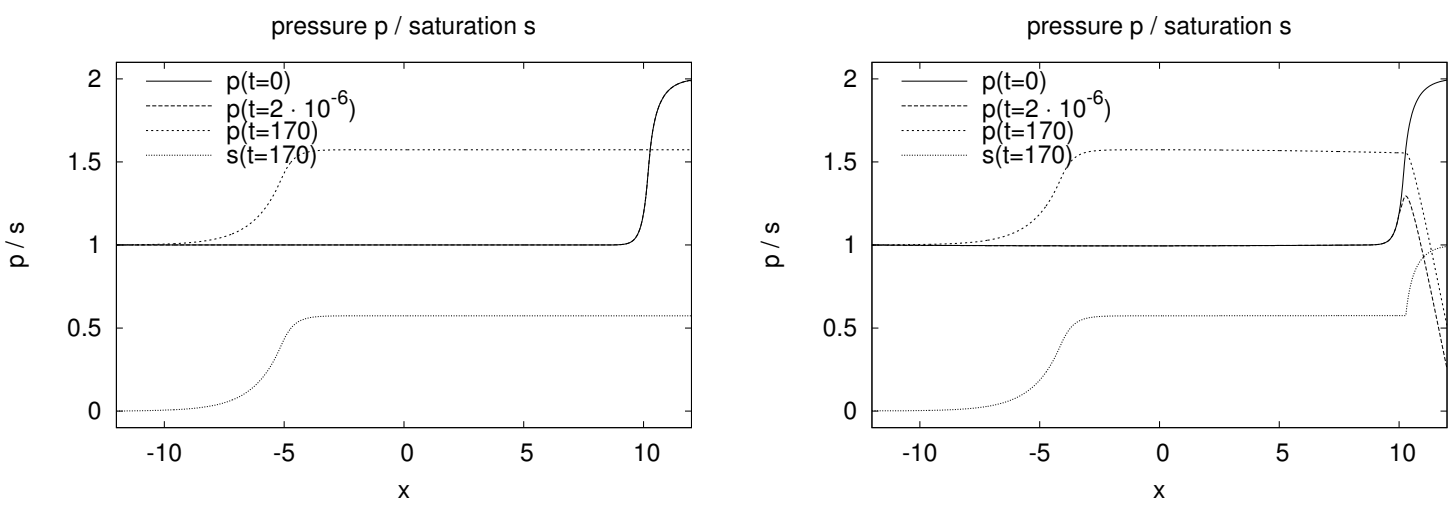

Figure 1: The effect of static hysteresis I. Pressure and saturation distribution in the one-dimensional case without dynamic term, $\tau=10^{-3}$. Both graphs show the pressure at times $t=0, t=2 \cdot 10^{-6}, t=170$, and the saturation at time $t=170$. Left: no static hysteresis, $\gamma=0, p_{c}(s)=s+1$. Right: with static hysteresis, $\gamma=1$, $p_{c}(s)=s$.

\subsection{With dynamic term, $\tau>0$}

We have seen already in the two-dimensional numerical experiments of $[7,8]$ that true fingering appears only for positive $\tau$. We illustrate in Figure 2 in one-dimensional calculations the effect of $\tau>0$. Except for this change, the parameters are as in Figure 1.

In the left graph we observe a well-known effect: the saturation profile in the finger is no longer monotone in $x$, but we observe a local maximum of the saturation at the finger-tip at about $x=-5$. Otherwise, the saturation profile and, accordingly, the pressure profile, is very similar to that of Figure 1, left.

A drastic change in the pressure profile becomes visible if we combine the effect of static hysteresis with the dynamic term, see Figure 2, right. Due to a positive $\tau$, the saturation inside the finger is not necessarily increasing for the whole time (it is even decreasing if local maxima are generated). This means that the static hysteresis term $\gamma \operatorname{sign}\left(\partial_{t} s\right)$ becomes relevant. Indeed, for $\partial_{t} s=0$ we find $\gamma \operatorname{sign}\left(\partial_{t} s\right)=[-\gamma, \gamma]$ and the hysteresis law generates a certain freedom for the pressure. Relation (1.5) allows the pressure to deviate largely from the imbibition value $p_{c}(s)+\gamma$. We observe in the right of Figure 2 that, indeed, the pressure assumes a completely different profile in the finger at time $t=170$ : it increases from the value -0.4 at the finger-root to the value 1.7 at the finger-tip. According to the hysteresis law, the saturation remains unchanged in the finger. We note that a non-monotone profile is not observed here; 

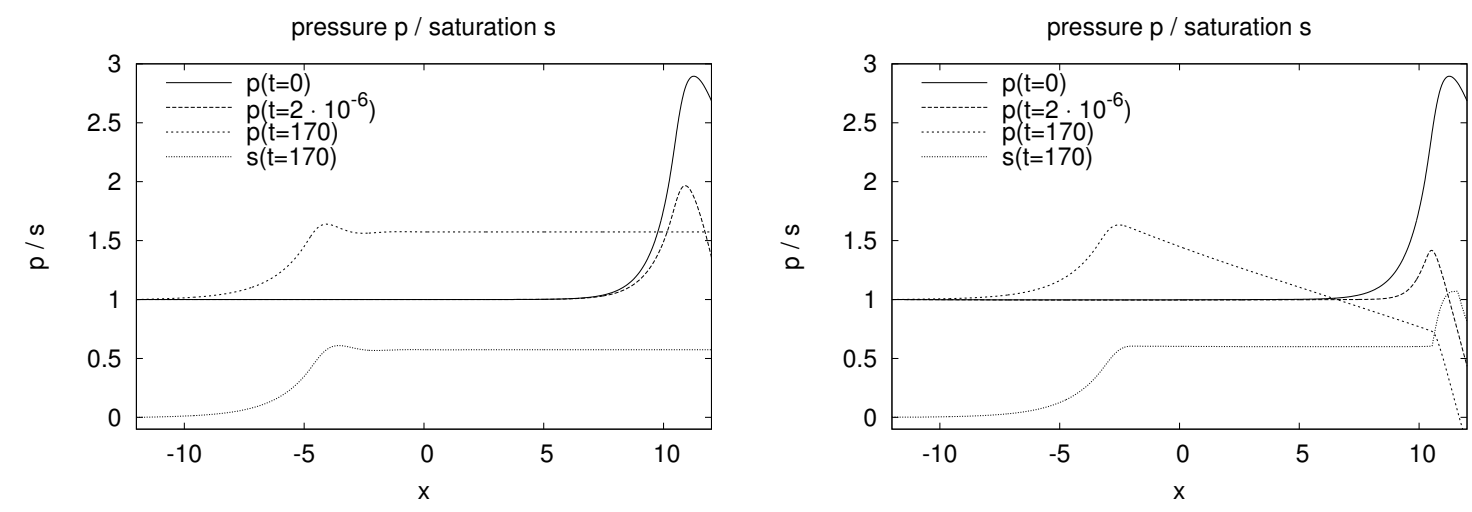

Figure 2: The effect of static hysteresis II. Pressure and saturation distribution in the one-dimensional case with dynamic term, $\tau=5$, other parameters as in 1 . Left: without static hysteresis. Right: with static hysteresis.

the value $\gamma=1$ is chosen large compared to the saturation decay behind the finger-tip and $\partial_{t} s=0$ holds in the entire bulk of the finger.

The pressure profile of Figure 2 is crucial for the explanation of fingering in the hysteresis model. The combined effect of hysteresis and $\tau$-term is that $\partial_{t} s=0$ and low pressures are possible inside the finger. A low pressure in the top part of the finger has the result that diffusion is of no importance and fingers are prevented from being smeared out. We present in Section 4 numerical experiments in a two-dimensional setting. They show the fingering effect. Our research interest here was to check the importance of static hysteresis for fingering. The result is that true fingering occurs only in the model where static hysteresis is combined with a positive $\tau$, in accordance with the above considerations.

\section{Solution concepts and existence results}

\subsection{Assumptions on data and the solution concept}

We describe first our assumptions on the coefficient functions. We emphasize that we always assume a strict positivity of the permeabilities and hence restrict ourselves to the non-degenerate case. Furthermore, we consider a saturation variable $s \in \mathbb{R}$ and do not impose a priori that the saturation values are between 0 and 1 . This fact is related to our assumption of a non-degenerate curve $s \mapsto p_{c}(s)$.

We assume with six positive numbers $K_{j}, \kappa_{j}, \kappa_{j}^{0}>0, j=1,2$

$$
\begin{aligned}
& p_{c} \in C^{0,1}(\mathbb{R} \times \Omega, \mathbb{R}), \quad \gamma \in C^{0,1}(\Omega,[0, \infty)), \\
& k_{j} \in C\left(\mathbb{R} \times \Omega,\left[\kappa_{j}, \kappa_{j}^{0}\right]\right),\left\|k_{j}(x, .)\right\|_{\operatorname{Lip}(\mathbb{R}, \mathbb{R})} \leq K_{j}, \text { for } j \in\{1,2\}, x \in \Omega .
\end{aligned}
$$

We furthermore make the mild additional assumption that $p_{c}(., x)$ has a non-negative primitive $P_{c} \in C(\mathbb{R} \times \Omega, \mathbb{R})$.

In our description of analytical result, we do not attempt to be precise in all assumptions on the data. Regarding initial and boundary conditions we only mention that an initial saturation is given by $s_{0} \in L^{2}(\Omega)$, that the boundary of the Lipschitz 
domain is decomposed in two different ways as $\partial \Omega=\bar{\Gamma}_{1} \cup \bar{\Sigma}_{1}=\bar{\Gamma}_{2} \cup \bar{\Sigma}_{2}$, that homogeneous Neumann conditions for fluid $j$ are imposed on $\Gamma_{j}$, and that Dirichlet data on $\Sigma_{j}$ are given by $p_{0,1}, p_{0,2} \in L^{2}\left(0, T ; H^{1}(\Omega)\right)$.

Solution concept. It turns out to be convenient to formulate the solution concept in a weak form. It can easily be verified that every function which is a weak solution of the conservation law and a pointwise solution of the play-type hysteresis law (1.5) also satisfies the energy inequality in 3. of Definition 3.1. Vice versa, for every weak solution of the conservation law satisfying also the pointwise inequality of 2., the energy inequality of 3. implies that also the play-type hysteresis law (1.5) is satisfied.

Definition 3.1 (Variational weak solution). Let $\left(s, p_{1}, p_{2}\right)$ be a triple of functions with

$$
s \in L^{\infty}\left(0, T ; L^{2}(\Omega)\right), \quad \partial_{t} s \in L^{2}\left(0, T ; L^{2}(\Omega)\right), \quad p_{1}, p_{2} \in L^{2}\left(0, T ; H^{1}(\Omega)\right),
$$

satisfying, in the sense of traces, initial and boundary conditions. The triple is called $a$ variational weak solution of the two-phase equation with hysteresis if the following three conditions are satisfied.

1. The evolution equations (1.1)-(1.2) and the no-flux conditions are satisfied in the weak sense.

2. The relation $p_{1}(x, t)-p_{2}(x, t)-p_{c}(s(x, t), x)-\tau \partial_{t} s(x, t) \in[-\gamma(x), \gamma(x)]$ holds for almost every $(x, t) \in \Omega_{T}$.

3. There holds the energy inequality

$$
\begin{aligned}
0 & \geq \int_{\Omega_{T}}\left(p_{c}(s)-p_{0,1}+p_{0,2}\right) \partial_{t} s+\int_{\Omega_{T}}\left\{\tau\left|\partial_{t} s\right|^{2}+\gamma\left|\partial_{t} s\right|\right\} \\
& +\int_{\Omega_{T}} k_{1}(s)\left[\nabla p_{1}+g_{1}\right] \nabla\left[p_{1}-p_{0,1}\right]+\int_{\Omega_{T}} k_{2}(s)\left[\nabla p_{2}+g_{2}\right] \nabla\left[p_{2}-p_{0,2}\right] .
\end{aligned}
$$

An analogous solution concept is used for the Richards equation. It can be shown that variational weak solutions are also weak solutions in a classical sense; we refer to [8] for this equivalence in the case of the Richards equation and to [7] for the equivalence in the two-phase flow system.

\subsection{Existence result}

The main existence result for the two-phase flow system with hysteresis is the following result of [7].

Theorem 3.2 (Existence result for two-phase flow). Let $\Omega \subset \mathbb{R}^{n}$ be polygonal, let $T>0$ and $\tau>0$ be positive, and let coefficient functions $p_{c}, k, \gamma$ be as in (3.1)-(3.2). Then there exists a weak variational solution $(s, p)$ of the hysteresis system. 
The existence proof is constructive in the following sense. We provide in [7] a Galerkin approximation of the hysteresis system, show the existence of solutions to the discrete system and the convergence of the approximate solutions to a solution of the hysteresis limit system.

Similar results have been obtained in [8] for the Richards equation. The assumptions on the coefficient functions and on the other data are identical. The main analytical tool for the existence proof is a compactness lemma that has first been shown in [8]. It provides the compactness of a family of functions $s$, when this family is obtained with the hysteresis law (1.5) from a family of pressure functions $p$ that possess additional spatial regularity.

\section{Calculations of gravity driven wetting fronts}

In this section, we present numerical results for finite element discretizations of the Richards equation and the two-phase flow system. The numerical schemes are described in more detail in [8] and [7], respectively. For other numerical approaches and detailed numerical analysis we refer to [12] and the references therein. For the inverted hysteresis relation (1.6), we use a regularized function $\Psi_{\gamma, \tau}^{\delta}: \mathbb{R} \rightarrow \mathbb{R}$,

$$
\Psi_{\gamma, \tau}^{\delta}=\Psi_{\delta, \tau}^{\delta}(z)= \begin{cases}\frac{z-\gamma}{\tau} & \text { for } z>\gamma+\tau \delta \\ \left(\frac{\gamma}{\delta}+\tau\right)^{-1} z & \text { for } z \in[-(\gamma+\tau \delta), \gamma+\tau \delta] \\ \frac{z+\gamma}{\tau} & \text { for } z<-(\gamma+\tau \delta),\end{cases}
$$

where $\delta>0$ is a regularizing parameter. In all numerical examples, we use the permeabilities

$$
k_{1}(s)= \begin{cases}\kappa_{1} & \text { for } \quad s<a_{1} \\ \kappa_{1}+k_{0,1}\left(s-a_{1}\right)^{2} & \text { for } \quad s \geq a_{1}\end{cases}
$$

and

$$
k_{2}(s)= \begin{cases}\kappa_{2} & \text { for } \quad s>1-a_{2}, \\ \kappa_{2}+k_{0,2}\left(\left(1-a_{2}\right)-s\right)^{2} & \text { for } \quad s \leq 1-a_{2}\end{cases}
$$

with $a_{1}, a_{2} \in[0,1]$ and $\kappa_{1}, \kappa_{2}, k_{0,1}, k_{0,2}>0$. Furthermore, we assume a van Genuchten type relation

$$
p_{c}(s)= \begin{cases}\alpha_{+}\left(\frac{1}{1-a_{2}-s+\varepsilon_{p}}-\frac{1}{1-a_{2}-a_{1}+\varepsilon_{p}}\right) & \text { for } s>a_{1}, \\ \alpha_{-}\left(s-a_{1}\right) & \text { for } s \leq a_{1},\end{cases}
$$

with $\alpha_{ \pm} \in \mathbb{R}$ and a small regularizing parameter $\varepsilon_{p}>0$. The vectors $g_{1}, g_{2} \in \mathbb{R}^{n}$ related to gravity forces are given by $g_{i}=\varrho_{i} e_{n}, i=1,2$, with densities $\varrho_{1}, \varrho_{2} \in \mathbb{R}$ and the $n$-th standard basis vector $e_{n} \in \mathbb{R}^{n}$.

In the following, we consider domains $\Omega:=(-L, L)^{n} \subset \mathbb{R}^{n}$ with $L>0$. With the definition $\Gamma_{ \pm}:=\left\{x \in \bar{\Omega}: x_{n}= \pm L\right\} \subset \partial \Omega$ and given functions $p_{1,-}, j_{2,-}$ : $\Gamma_{-} \times\left(t_{0}, T\right] \rightarrow \mathbb{R}$ and $j_{1,+}, p_{2,+}: \Gamma_{+} \times\left(t_{0}, T\right] \rightarrow \mathbb{R}$, we assume Dirichlet boundary conditions $p_{1}=p_{1,-}$ for $x \in \Gamma_{-}$and $p_{2}=p_{2,+}$ for $x \in \Gamma_{+}$and Neumann boundary 
conditions

$$
\begin{array}{ll}
j_{1}:=-k_{1}(s)\left(\nabla p_{1}+\varrho_{1} e_{n}\right) \cdot \nu_{+}=j_{1,+} & \text { for } \quad x \in \Gamma_{+}, \\
j_{2}:=-k_{2}(s)\left(\nabla p_{2}+\varrho_{2} e_{n}\right) \cdot \nu_{-}=j_{2,-} & \text { for } \quad x \in \Gamma_{-},
\end{array}
$$

$\nu_{ \pm}= \pm e_{n}$ denoting the outer normals to $\Gamma_{ \pm}$. In the lateral directions, i.e. for $x_{i} \in\{-L, L\}, i \in\{1, \ldots, n-1\}$, we assume periodic pressures $p_{1}$ and $p_{2}$.

At the upper boundary, we have used a time dependent flux condition

$$
j_{1,+}= \begin{cases}j_{1,+}^{0} & \text { for } t<t_{s} \\ j_{1,+}^{s} & \text { for } t \geq t_{s}\end{cases}
$$

with $t_{s}>t_{0}$ and $j_{1,+}^{0}, j_{1,+}^{s} \in \mathbb{R}$. On the lower boundary, we assume the constant flux

$$
j_{2,-} \equiv j_{2,-}^{0}
$$

for a given value $j_{2,-}^{0} \in \mathbb{R}$. The Dirichlet boundary conditions are

$$
p_{1,-} \equiv \gamma-\alpha_{-} a_{1}, \quad p_{2,+} \equiv p_{2,+}^{0}
$$

with a constant pressure $p_{2,+}^{0}$. In all numerical experiments, the space dimension is $n=2$. We study moderate deterministic perturbations of the initial condition $s=0$ of the form

$$
s_{0}(x)=\sum_{i=1}^{10} A_{i}\left(1-\tanh \left(3\left(\left|x-x_{0, i}\right|-1 / 2\right)\right)\right),
$$

where $x_{0, i}=\left(-L+\frac{(2 i-1)}{10} L, 23.5\right), i=1, \ldots, 10$ and amplitudes $A_{1}=0.6, A_{2}=0.4$, $A_{3}=0.2, A_{4}=0.5, A_{5}=0.2, A_{6}=0.3, A_{7}=0.1, A_{8}=0.7, A_{9}=0.5, A_{10}=0.1$. In other experiments we use random initial data of the form

$$
s_{0}(x)=0.001+\mathcal{R}(x)
$$

where $\mathcal{R}(x) \in[-0.001,0.001]$ is a space dependent random number.

We provide a list of all other parameters in Table 1. Note that we switch off hysteresis by setting $\gamma=0$, this also affects the boundary condition at the bottom of the computational domain.

\begin{tabular}{|c|c|c|c|c|c|c|c|c|c|c|c|}
\hline parameter & $\gamma$ & $\kappa_{1}$ & $\kappa_{2}$ & $k_{0,1}$ & $k_{0,2}$ & $\delta$ & $\tau$ & $a_{1}$ & $a_{2}$ & $t_{s}$ & $t_{0}$ \\
\hline value & $4 ; 0$ & $10^{-4}$ & $10^{-2}$ & 1 & 0.1 & $10^{-7}$ & 0.5 & 0.32 & 0 & 0 & -2 \\
\hline \hline parameter & $\varrho_{1}$ & $\varrho_{2}$ & $\varepsilon_{p}$ & $\alpha_{+}$ & $\alpha_{-}$ & $j_{1,+}^{0}$ & $j_{1,+}^{s}$ & $p_{2,+}^{0}$ & $j_{2,-}^{0}$ & $L$ & \\
\hline value & 1 & 0 & $10^{-10}$ & 0.1 & 50 & 0.524 & 0.01 & 0 & 0 & 24 & \\
\hline
\end{tabular}

Table 1: Parameters used for numerical results. 


\subsection{Richards equation}

We investigate the Richards equation in two settings in order to show the effect of perturbations of the initial data. In the numerical experiments of Figure 3, we show the solutions at different time instances for initial values which are perturbed from a uniform setting in a deterministic way with moderate perturbations. The perturbations generate fingers at prescribed positions, but we note that fingers may cease to grow even at very small times.

The upper row shows the evolution with static hysteresis, the lower row the evolution without static hysteresis. We see that, without static hysteresis, the fingers have the tendency to merge in the process and, as a result, no true fingers are visible.

From the numerical results, we calculate additionally a measure for the typical pressure gradient between upper and lower boundary, setting

$$
d_{p}=\frac{1}{2 L} \int_{-L}^{L}\left(p_{h}\left(x_{1}, L, t\right)-p_{h}\left(x_{1},-L, t\right)\right) \mathrm{d} x_{1}
$$

Since the flux is prescribed, a larger pressure gradient indicates a smaller effective permeability of the medium and vice versa. The calculated values for $d_{p}$ show that the generation of fingers increases the effective permeability of the medium.

Figure 4 shows solutions to the same model, just that we now use small, random perturbations of the initial data. We observe that more fingers are generated. The qualitative result of the deterministic perturbation experiment remains valid: only the combination of hysteresis and $\tau>0$ generates fingers.

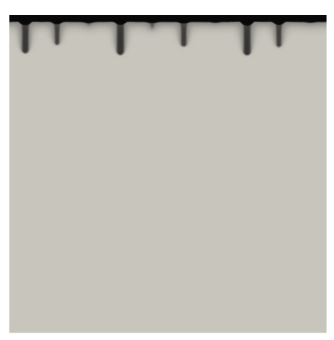

$d_{p} \approx 13.61$

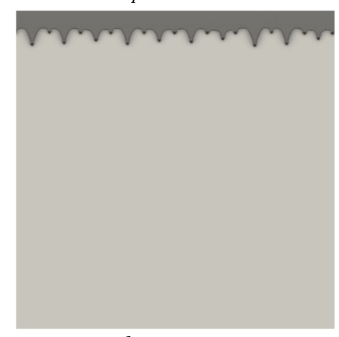

$d_{p} \approx 16.03$

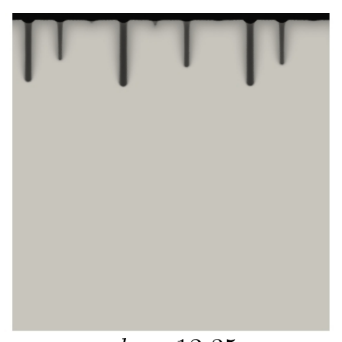

$d_{p} \approx 12.35$

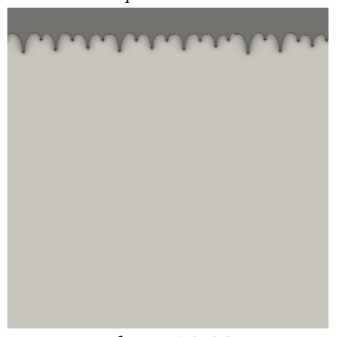

$d_{p} \approx 16.03$

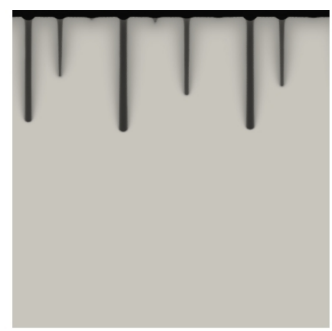

$d_{p} \approx 10.72$

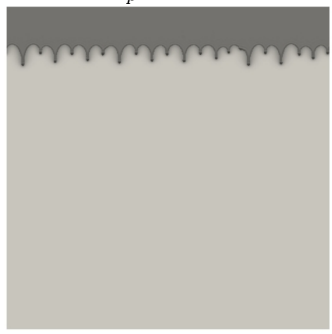

$d_{p} \approx 16.03$

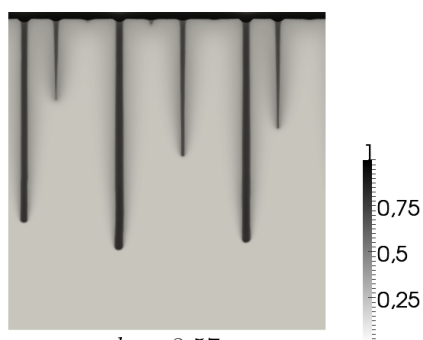

$d_{p} \approx 8.57$

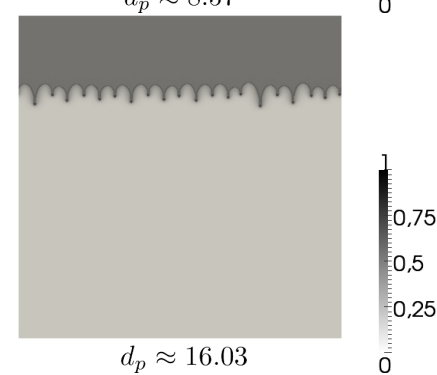

Figure 3: With and without static hysteresis, Richards equation, I. The figure illustrates the time evolution for $\tau=0.5$, the initial values include a deterministic perturbation Both rows indicate saturation values at times $t \approx 56, t \approx 114, t \approx 201$, and $t \approx 406$. Upper row: evolution with static hysteresis, $\gamma=4$. Lower row: evolution without static hysteresis, $\gamma=0$. 


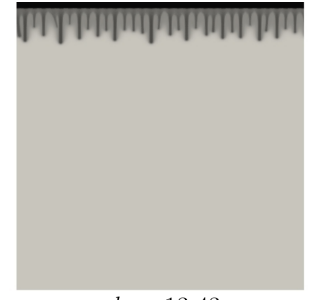

$d_{p} \approx 13.43$

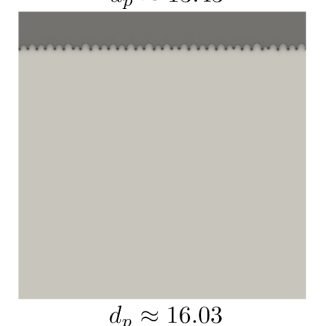

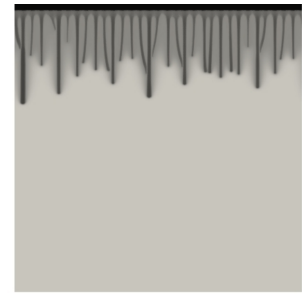

$d_{p} \approx 12.00$

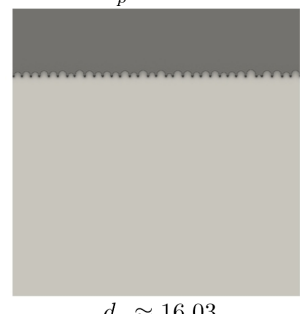

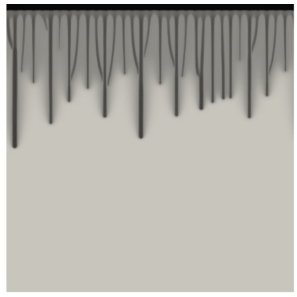

$d_{p} \approx 11.18$

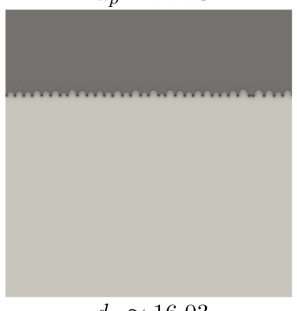

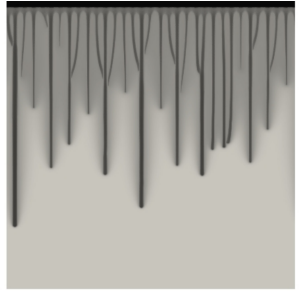

$d_{p} \approx 10.17$

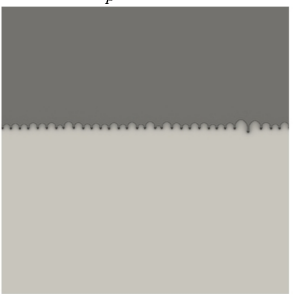

$d_{p} \approx 16.03$
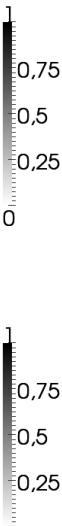

Figure 4: With and without static hysteresis, Richards equation, II. The figure illustrates the time evolution of saturation values with $\tau=0.5$, the initial values include a small random perturbation. Upper row: evolution with static hysteresis at times $t \approx 171, t \approx 389, t \approx 536, t \approx 798$. Lower row: evolution without static hysteresis at times $t \approx 172, t \approx 394, t \approx 530, t \approx 792$.

\subsection{Two-phase flow equation}

The experiments have also been performed for the two-phase flow equation. The qualitative finding are exactly as for the Richards equation. The indicated pressure difference $d_{p_{1}}$ now refers to pressure differences in the first fluid. Figure 5 shows the results for a small, random perturbation of the initial data.

In comparison to the Richards equation we observe slightly thicker fingers. Even in the model without static hysteresis we find, locally along the front, a structure that resembles a fingering effect. In this sense, we see that introducing the second phase in the model makes the fingering more pronounced.

\section{References}

[1] F. Bagagiolo and A. Visintin. Hysteresis in filtration through porous media. Z . Anal. Anwendungen, 19(4):977-997, 2000.

[2] T. W. J. Bauters, D. A. DiCarlo, T. S. Steenhuis, and J.-Y. Parlange. Soil water content dependent wetting front characteristic in sands. Elsevier Journal of Hydrology, 231-232:244-254, 2000.

[3] A. Y. Beliaev and S. M. Hassanizadeh. A theoretical model of hysteresis and dynamic effects in the capillary relation for two-phase flow in porous media. Transp. Porous Media, 43(3):487-510, 2001.

[4] J. Carrillo and P. Wittbold. Uniqueness of renormalized solutions of degenerate elliptic-parabolic problems. J. Differential Equations, 156(1):93-121, 1999. 


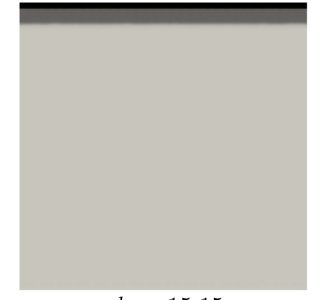

$d_{p} \approx 15.15$

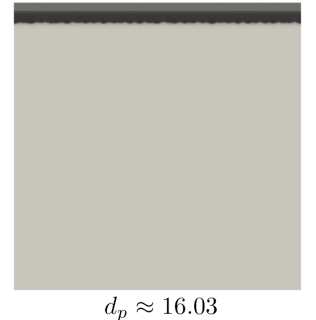

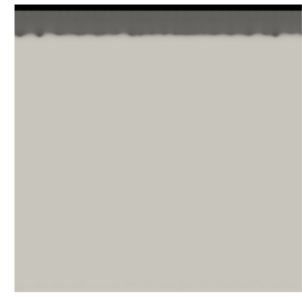

$d_{p} \approx 14.58$

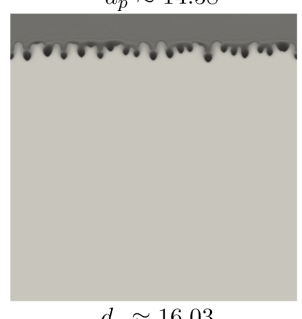

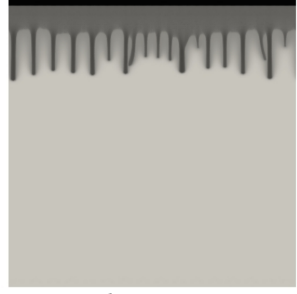

$d_{p} \approx 13.06$

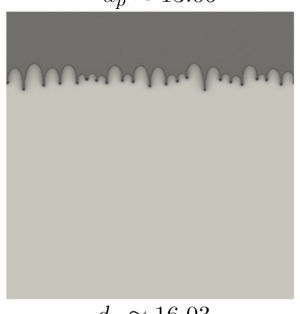

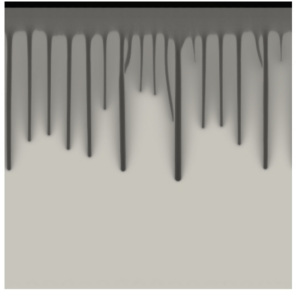

$d_{p} \approx 12.12$

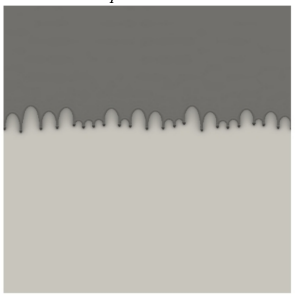

$d_{p} \approx 16.03$

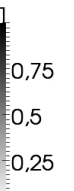

0

Figure 5: With and without static hysteresis, two-phase flow. The figure illustrates the time evolution of saturation values for $\tau=0.5$, the initial values include a small random perturbation. Upper row: evolution with static hysteresis at times $t \approx 125$, $t \approx 202, t \approx 405, t \approx 787$. Lower row: evolution without static hysteresis at times $t \approx 108, t \approx 208, t \approx 408, t \approx 786$.

[5] C. Cuesta, C. J. van Duijn, and J. Hulshof. Infiltration in porous media with dynamic capillary pressure: travelling waves. European J. Appl. Math., 11(4):381$397,2000$.

[6] A. G. Egorov, R. Z. Dautov, J. L. Nieber, and A. Y. Sheshukov. Stability analysis of gravity-driven infiltrating flow. Water Resources Research, 39(9):12-1-12-14, 2003.

[7] J. Koch, A. Rätz, and B. Schweizer. Two-phase flow equations with a dynamic capillary pressure. Preprint TU Dortmund, 2011.

[8] A. Lamacz, A. Rätz, and B. Schweizer. A well-posed hysteresis model for flows in porous media and applications to fingering effects. Adv. Math. Sci. Appl., 21(1), 2011.

[9] J. L. Nieber, T. W. J. Bauters, T. S. Steenhuis, and J.-Y. Parlange. Numerical simulation of experimental gravity-driven unstable flow in water repellent sand. Elsevier Journal of Hydrology, 231-232:295-307, 2000.

[10] J. L. Nieber, R. Z. Dautov, A. G. Egorov, and A. Y. Sheshukov. Dynamic capillary pressure mechanism for instability in gravity-driven flows; review and extension to very dry conditions. Transp Porous Med, 58:147-172, 2005.

[11] F. Otto. $L^{1}$-contraction and uniqueness for unstationary saturated-unsaturated porous media flow. Adv. Math. Sci. Appl., 7(2):537-553, 1997.

[12] F. A. Radu, I. S. Pop, and P. Knabner. Error estimates for a mixed finite element discretization of some degenerate parabolic equations. Numer. Math., 109(2):285-311, 2008. 
[13] B. Schweizer. Laws for the capillary pressure in a deterministic model for fronts in porous media. SIAM J. Math. Anal., 36(5):1489-1521 (electronic), 2005.

[14] B. Schweizer. A stochastic model for fronts in porous media. Ann. Mat. Pura Appl. (4), 184(3):375-393, 2005.

[15] B. Schweizer. Instability of gravity wetting fronts for richards equations with hysteresis. Interfaces and Free Boundaries (accepted), 2012.

[16] J. S. Selker, J.-Y. Parlange, and T. S. Steenhuis. Fingered flow in two dimensions. part 2. predicting finger moisture profile. Wat. Resources Res., 28(9):2523-2528, 1992.

[17] C. J. van Duijn, L. A. Peletier, and I. S. Pop. A new class of entropy solutions of the Buckley-Leverett equation. SIAM J. Math. Anal., 39(2):507-536 (electronic), 2007.

[18] C. J. van Duijn, G. J. M. Pieters, and P. A. C. Raats. Steady flows in unsaturated soils are stable. Transp. Porous Media, 57(2):215-244, 2004. 


\section{Preprints ab 2009/16}

2012-02

2012-01

2011-14

$2011-13$

$2011-12$

2011-11

2011-10

2011-09

2011-08

2011-07

2011-06

2011-05

2011-04

2011-03

2011-02

2011-01

2010-16

Andreas Rätz and Ben Schweizer

Hysteresis models and gravity fingering in porous media

Wilfried Hazod

Intrinsic topologies on $\mathrm{H}$-contraction groups with applications to semistability

Guy Bouchitté and Ben Schweizer

Plasmonic waves allow perfect transmission through sub-wavelength metallic gratings

Waldemar Grundmann

Moment functions and Central Limit Theorem for Jacobi hypergroups on $[0, \infty[$

J. Koch, A. Rätz, and B. Schweizer

Two-phase flow equations with a dynamic capillary pressure

Michael Voit

Central limit theorems for hyperbolic spaces and Jacobi processes on $[0, \infty[$

Ben Schweizer

The Richards equation with hysteresis and degenerate capillary pressure

Andreas Rätz and Matthias Röger

Turing instabilities in a mathematical model for signaling networks

Matthias Röger and Reiner Schätzle

Control of the isoperimetric deficit by the Willmore deficit

Frank Klinker

Generalized duality for k-forms

Sebastian Aland, Andreas Rätz, Matthias Röger, and Axel Voigt

Buckling instability of viral capsides - a continuum approach

Wilfried Hazod

The concentration function problem for locally compact groups revisited: Non-dissipating space-time random walks, $\tau$-decomposable laws and their continuous time analogues

Wilfried Hazod, Katrin Kosfeld

Multiple decomposability of probabilities on contractible locally compact groups

Alexandra Monzner* and Frol Zapolsky $\dagger$

A comparison of symplectic homogenization and Calabi quasi-states

Stefan Jäschke, Karl Friedrich Siburg and Pavel A. Stoimenov

Modelling dependence of extreme events in energy markets using tail copulas

Ben Schweizer and Marco Veneroni

The needle problem approach to non-periodic homogenization

Sebastian Engelke and Jeannette H.C. Woerner

A unifying approach to fractional Lévy processes 
2010-14 Lorenz J. Schwachhöfer

On the Solvability of the Transvection group of Extrinsic

Symplectic Symmetric Spaces

2010-13 Marco Veneroni

Stochastic homogenization of subdifferential inclusions via scale integration

2010-12 Agnes Lamacz, Andreas Rätz, and Ben Schweizer

A well-posed hysteresis model for flows in porous media and applications to fingering effects

2010-11 Luca Lussardi and Annibale Magni

$\Gamma$-limits of convolution functionals

2010-10 Patrick W. Dondl, Luca Mugnai, and Matthias Röger

Confined elastic curves

2010-09 Matthias Röger and Hendrik Weber

Tightness for a stochastic Allen-Cahn equation

2010-08 Michael Voit

Multidimensional Heisenberg convolutions and product formulas

for multivariate Laguerre polynomials

2010-07 Ben Schweizer

Instability of gravity wetting fronts for Richards equations with hysteresis

2010-06 Lorenz J. Schwachhöfer

Holonomy Groups and Algebras

2010-05 Agnes Lamacz

Dispersive effective models for waves in heterogeneous media

2010-04 Ben Schweizer and Marco Veneroni

Periodic homogenization of Prandtl-Reuss plasticity equations in arbitrary dimension

2010-03 Holger Dette and Karl Friedrich Siburg and Pavel A. Stoimenov

A copula-based nonparametric measure of regression dependence

2010-02 René L. Schilling and Alexander Schnurr

The Symbol Associated with the Solution of a Stochastic Differential Equation

2010-01 Henryk Zähle

Rates of almost sure convergence of plug-in estimates for distortion risk measures

2009-16 Lorenz J. Schwachhöfer

Nonnegative curvature on disk bundles 